\title{
Congenital bile acid synthesis defect type
} 3

INSERM

\section{Source}

INSERM. (1999). Orphanet: an online rare disease and orphan drug data base. Congenital bile acid synthesis defect type 3. ORPHA:79302

Congenital bile acid synthesis defect type 3 (BAS defect type 3 ) is a severe anomaly of bile acid synthesis (see this term) characterized by severe neonatal cholestatic liver disease. 